\title{
Simulation Analysis of Hierarchical Technique in Mobile Ad-hoc Network
}

\author{
Anzar Ahmad ${ }^{1}$, R.C Joshi ${ }^{2}$, S. C Gupta ${ }^{3}$ \\ Electronics \& Commuication ${ }^{1}$, Computer Science ${ }^{2}$,Electronics \& Communication ${ }^{3}$, \\ Graphic Era University ${ }^{1-3}$ 566/6 Bell Road, Clement Town, Dehradun(India) \\ 1 anz.hmd@gmail.com \\ ${ }^{3}$ sureshprem1938@gmail.com \\ *2 chancellor geu@gamil.com
}

\begin{abstract}
Mobile adhoc network(MANET) is penetrating our society as a future technology in our day to day life. Today mobile ad-hoc network has lot of application like in disaster management, tactical network, sensor networks etc. But its implementation for commercial purposes is not an easy as compare to other exiting wireless technologies such as cellular networks. It has many challenges or issues that offer many problems concerning mobility managements, shared radio medium, routing protocol and scalability. To overcome such issues many approaches has been described. Clustering schemes are one of them in order to manage/organize the larger network in hierarchical manner. So many clustering techniques have been developed. Clustering is a scheme which divide large network into small manageable networks or group. These groups are called clusters. Clustering increases network capacity and reduces delay and the routing overheads and make the network manageable and efficient in the presence of high mobility and large number of nodes. In hierarchical network every cluster has a group leader node which has many responsibility such as e to manage that cluster, routing table updates, and the discovery of new routes within the network. The other node named as gateway node is responsible for inter cluster communication. But the frequent change of cluster leader and gateway node leads loss of routing information stored, inefficient performance of routing protocols, more delays, more overheads and makes the network unstable. In the proposed approach in place of cluster leader and gate way node, a new node named as Cluster leader gateway(CLG) node id selected and all of the taskof cluster leader and gateway node is performed by the this single CLG node itself . The propose CLG approach is simulated and compare with existing approach the help of Exata Cyber++ simulator and performs better for the parameter end to end delay,jitter,number of packets drop and link utilization.
\end{abstract}

Keyword- MANET, MANET, cluster, hierarchical, CLG, delays, Overheads,

\section{INTRODUCTION}

Today wireless technology is touching every aspect of our life and has become integral part of our daily lives. It is becoming everyone favourite with the use of transportable wireless devices like mobile phone notebook, tablet and fablet In organize to make use of these devices some type of fixed communications is needed such as access points or base stations which has a limited premises or range. Mobile ad hoc networks (MANET) is a solution to such kinds of problems. MANETs are self-configured, independent infrastructure less networks having movable nodes that are linked by multi-hop wireless links [9]. The basic idea of a MANET is that a network can be establish without the need for any central manager or fixed infrastructure. MANET presents many challenges to the research society because of vibrant topologies. In addition, connection bandwidth and mobile nodes transmission power are also limited. Scalability is of particular attention to ad hoc network designer and user and is a problem with important influence on capability and capacity. The scalability concern of MANET is address through a hierarchical approach that partitions the network into cluster. A cluster is basically a subset of nodes of the network that satisfies a certain property. Clusters are equivalent to cells in a cellular network. In this way, the network becomes more convenient [5][7]. It must be understandable though that a clustering technique is not a routing protocol. Clustering is a technique which aggregate nodes into groups. These groups are contained by the network and they are known as clusters. A cluster is basically a subset of nodes of the network that satisfies a certain property [15]. Each cluster has some nodes and a cluster leader which come across after other nodes in that cluster and a gateway node which communicate to another cluster in the same network. In this way, the network becomes more convenient [14]

\section{RELATED WORK}

Scalability is one of the major challenges in mobile ad-hoc networks. Presently ad hoc network applications occupy huge network with thousands number of nodes which are difficult to handle. Clustering is a first-class technique to divide big network into small network [9] in MANET as it reduces end to end delay, overheads and make the network more controllable in the presence of huge numbers of nodes and high mobility. Nodes are very close to each other generally one hop or two hop away. Each cluster has one or more gateway terminal to connect to another cluster in the network [9]. Back bone base routing [13] and spine based routing [14], uses a similar 
approach. Clustering provide many reward for the medium access layer and the network layer in MANET. The realization of clustering technique allows a improved performance of the protocols for the MAC layer by improving the frequency reuse, end to end delay, jitter, throughput and power consumption. Also clustering help to get better routing at the network layer by falling the size of the routing tables and by lessening transmission overhead due to the bring up to date of routing tables after topological change occur [16] [17]. Clustering help collective topology information since the number of nodes of a cluster is smaller than the number of nodes of the whole network [14] [15]. Therefore, each terminal only wants to store up a fraction of the total network routing information [22]. In clustering technique nodes are mandatory to play different role per their capacity or potential as criteria. In common, three types of nodes are defined [18]. Normal nodes are member of a cluster which do not have neighbours belong to a different cluster.

Gateway nodes are nodes in a non-cluster head state positioned at the edge of a cluster.

Many clustering approaches for mobile ad hoc networks choose a split of nodes to shape a network backbone that supports control function. A set of the chosen nodes are called cluster Leader (CL) and each node in the network is linked with one. Cluster heads are associated with one another directly or through gateway nodes [4]. The combination of gateway nodes and cluster heads form a associated backbone. This connected backbone helps make simpler functions such as channel access, bandwidth allocation, routing power control and virtual-circuit support. To achieve this, a gateway node must have at least one neighbour that is a member of another cluster.

\section{PROPOSED APPROACH}

In proposed approach we compared hierarchical network for existing clustering scheme which has a cluster Leader(CL) and gateway node which is exposed in Fig. 1 and cluster Leader gateway(CLG) approach in which in place of cluster leader and gateway node, a CLG node acts as a cluster leader as well as gateway exposed in Fig.2

\section{PROBLEM DEFINITION}

In In present simulation work, we have taken total 105 mobile nodes each allow with AODV routing protocol, and all nodes are randomly distributed with the speed of $0-10 \mathrm{~m} / \mathrm{s}$ and all nodes sending CBR file. Environment size for simulation is $1500 \times 1500$ metres.

\section{Simulation Setup:}

The simulation parameter are as follows:

TABLE 1 : Simulation parameter

\begin{tabular}{|l|l|l|}
\hline S.N & Parameter & value \\
\hline 1 & Environment size & $(1500 \times 1500) m w t e r$ \\
\hline 2 & Total number of nodes & 105 \\
\hline 3 & File type & CBR \\
\hline 4 & Speed of the node & $(0-10) \mathrm{m} / \mathrm{s}$ \\
\hline 5 & Start time & $10 \mathrm{sec}$ \\
\hline 6 & Pause Time & $0 \quad$ sec \\
\hline
\end{tabular}

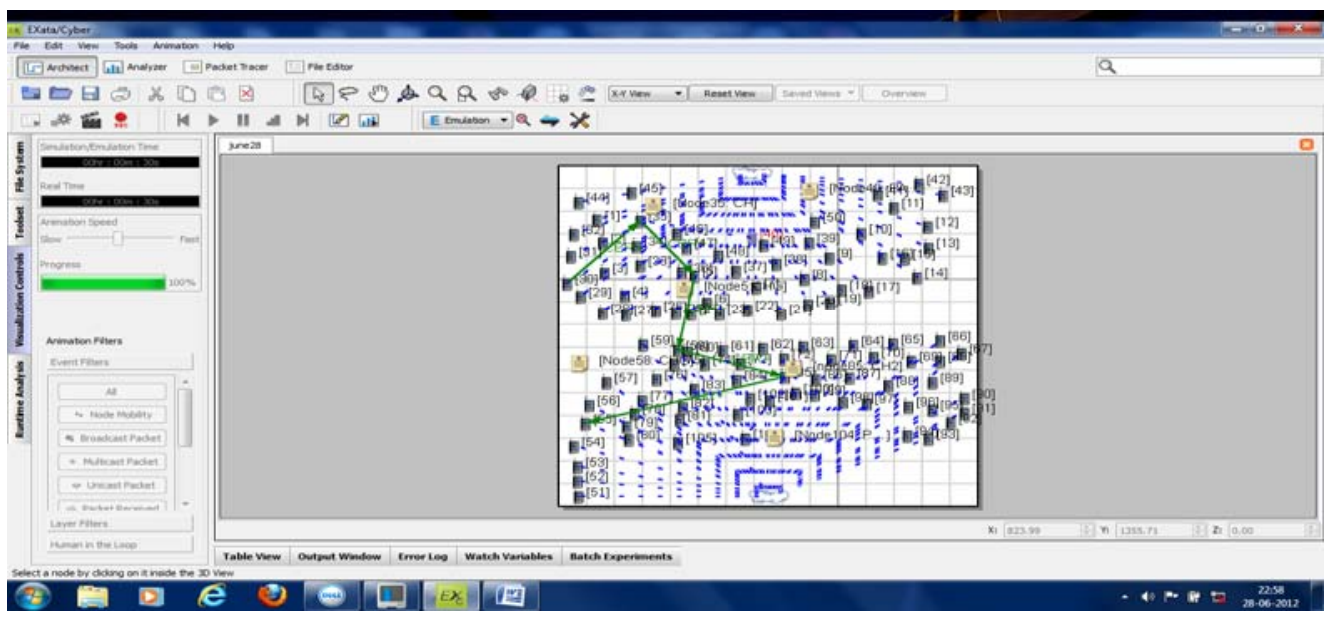

Fig.1. CL\& gateway node scenario 


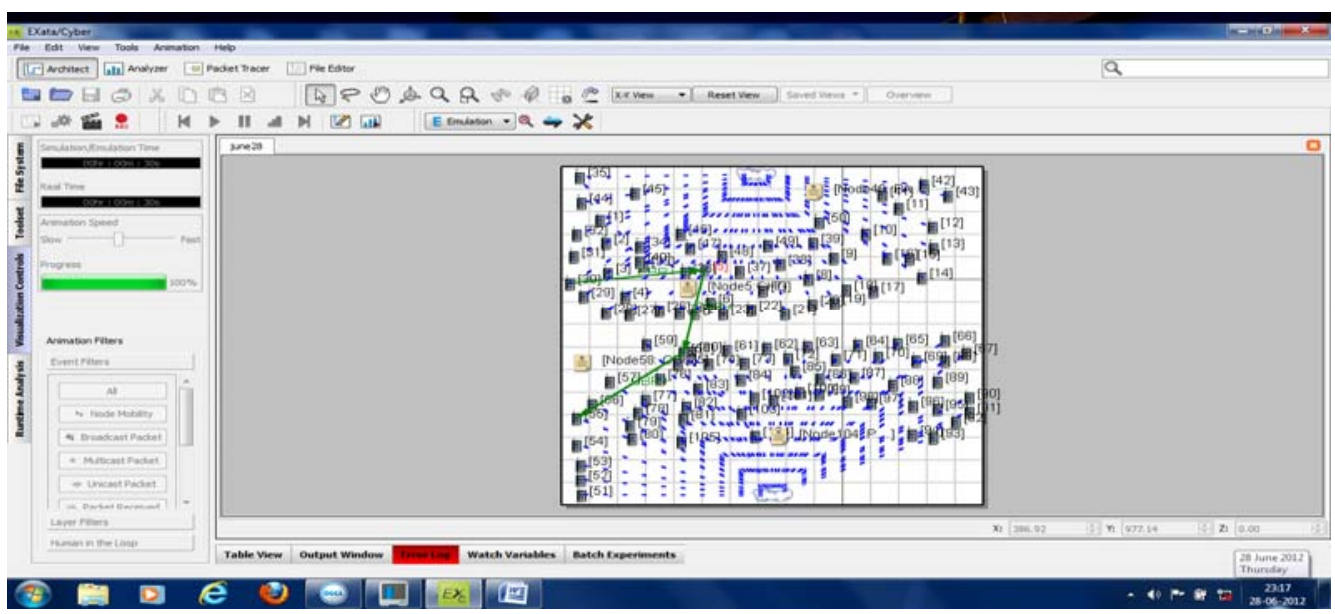

Fig.2. CLG scenario

\section{SIMULATION AND RESULT DISCUSSION}

We used Exata Cyber simulator to simulate CL \& G and CLG approach. EXata is a complete suite of tools for emulating large wired and wireless networks. It is used to simulate and emulate to find the behaviour and performance of networks to improve their design, operation, and management. It also provides a cost-effective and easy-to-use alternative to physical test beds that typically have high equipment costs, complex setup requirements and limited scalability. It creates a digital network replica that interfaces with real networks and applications.

\section{Performance Analysis for end-to end delay in CH \& G and CHG Approach}

In the result analysis of CBR server with cluster leader \& gateway node, and Cluster leader gateway (CLG) approach the end to end delay for the CLG is a better for as compare to L and G approach as shown in Fig. 3 and Fig.4

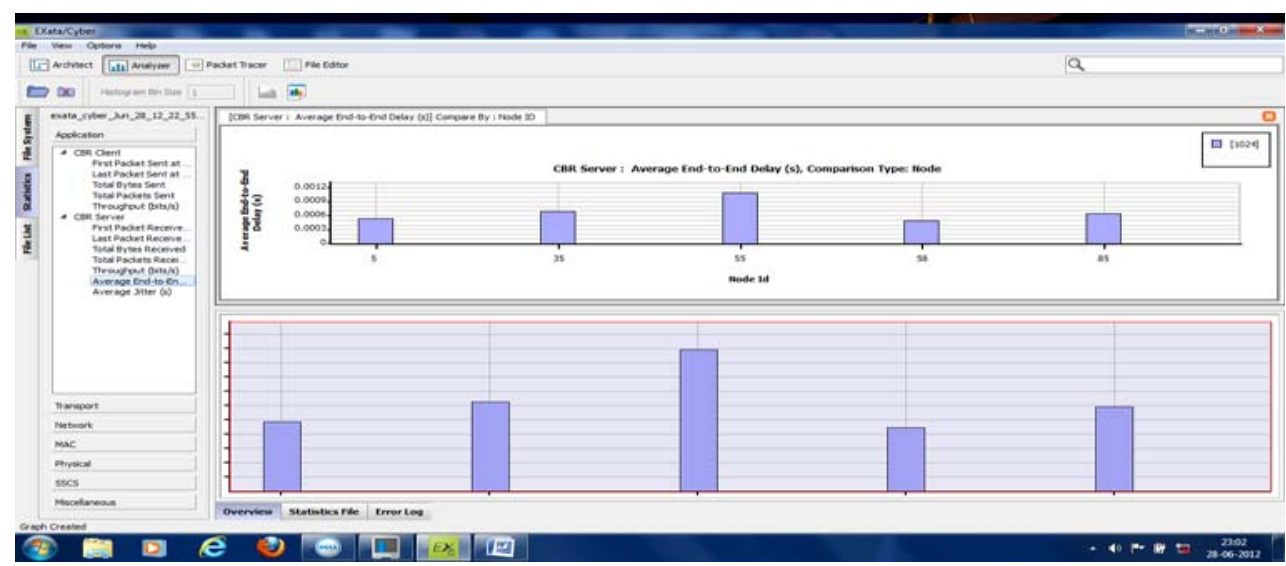

Fig. 3. Average end to end delay for CL\& G

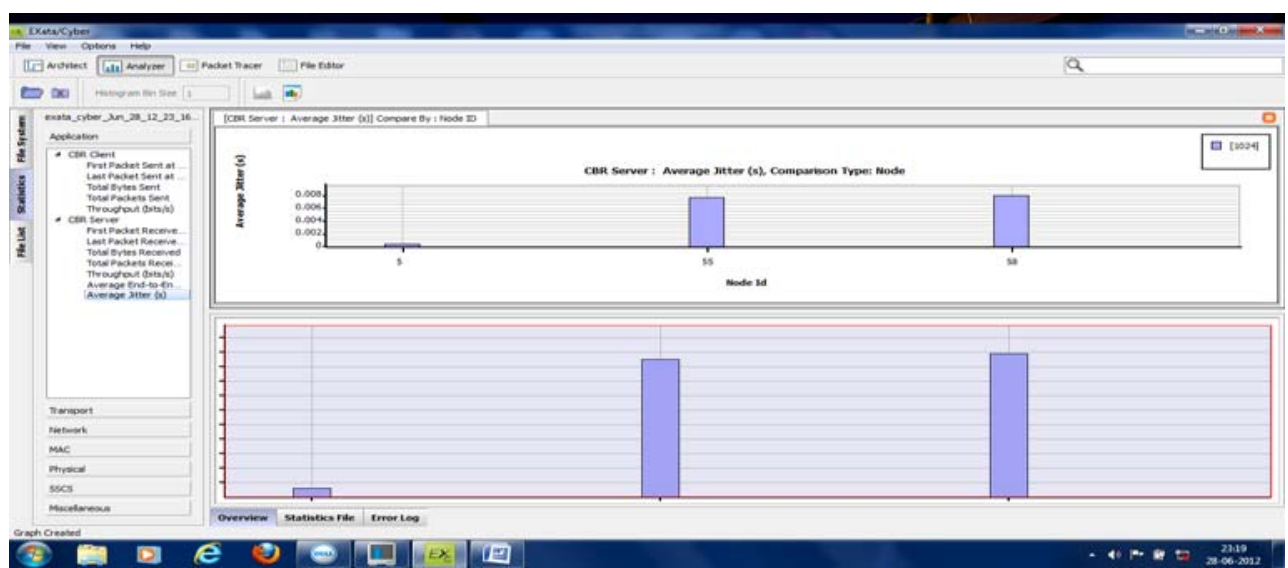

Fig. 4. Avg end to end delay in CL G 


\section{Performance Analysis for Average Jitter in CL \& G and CLG Approach}

In the result analysis of CBR server with cluster leader \& gateway, and Cluster leader gateway (CLG) the average jitter is less in CLG approach as shown in Fig.5 and Fig.6.

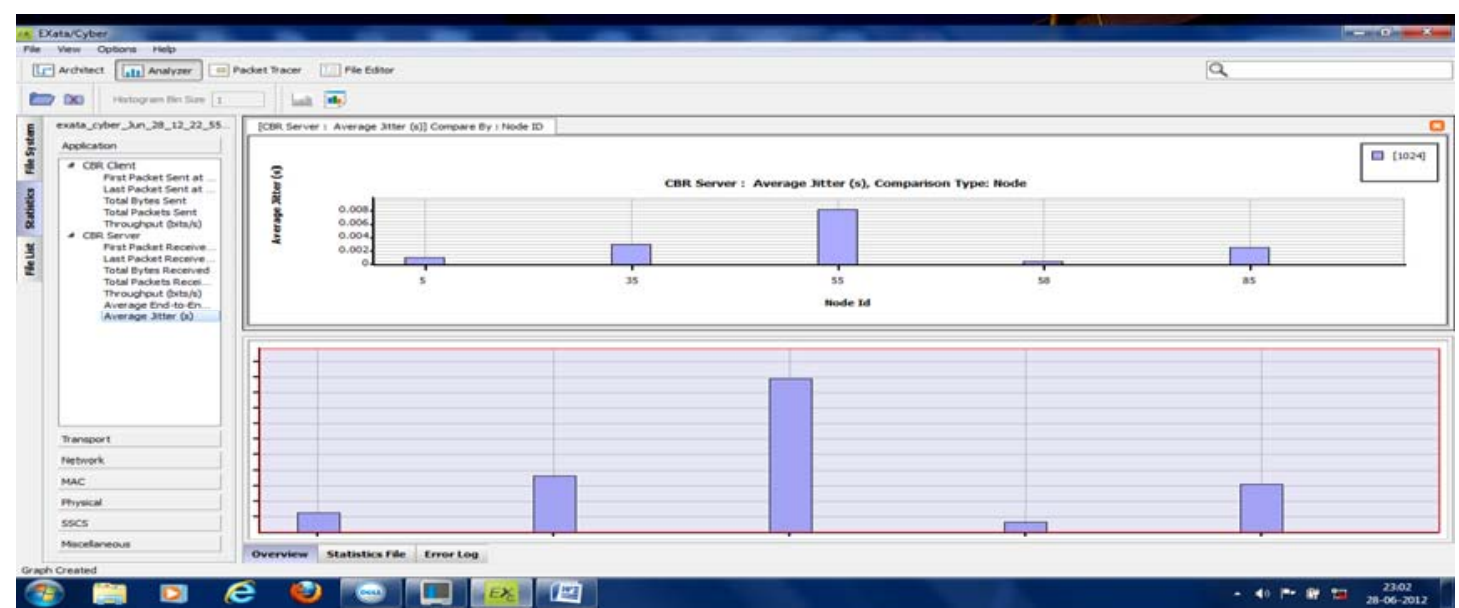

Fig5 : Average jitter CL \& G

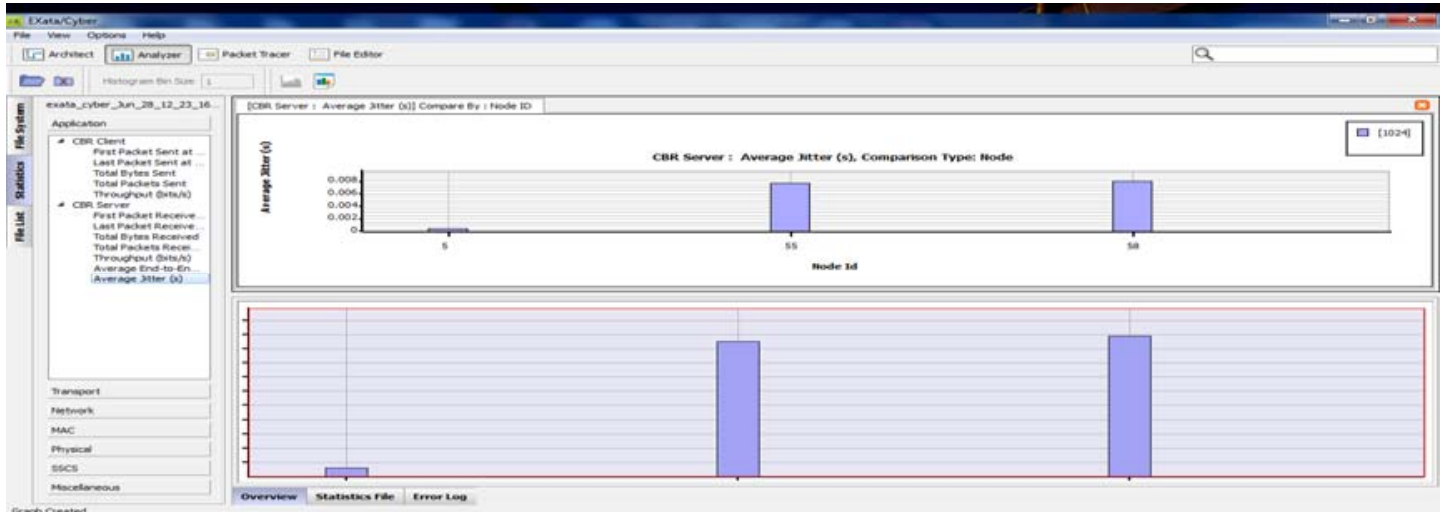

Fig6: Average jitter in CLG

Performance Analysis for number of packets dropped in CH \& G and CHG Approach

In the result analysis of CBR server with cluster leader \& gateway, and Cluster leader gateway (CLG) the number of packets dropped in CLG approach is less and overall performance of the network is very much improved as shown in the Fig.7 and Fig.8

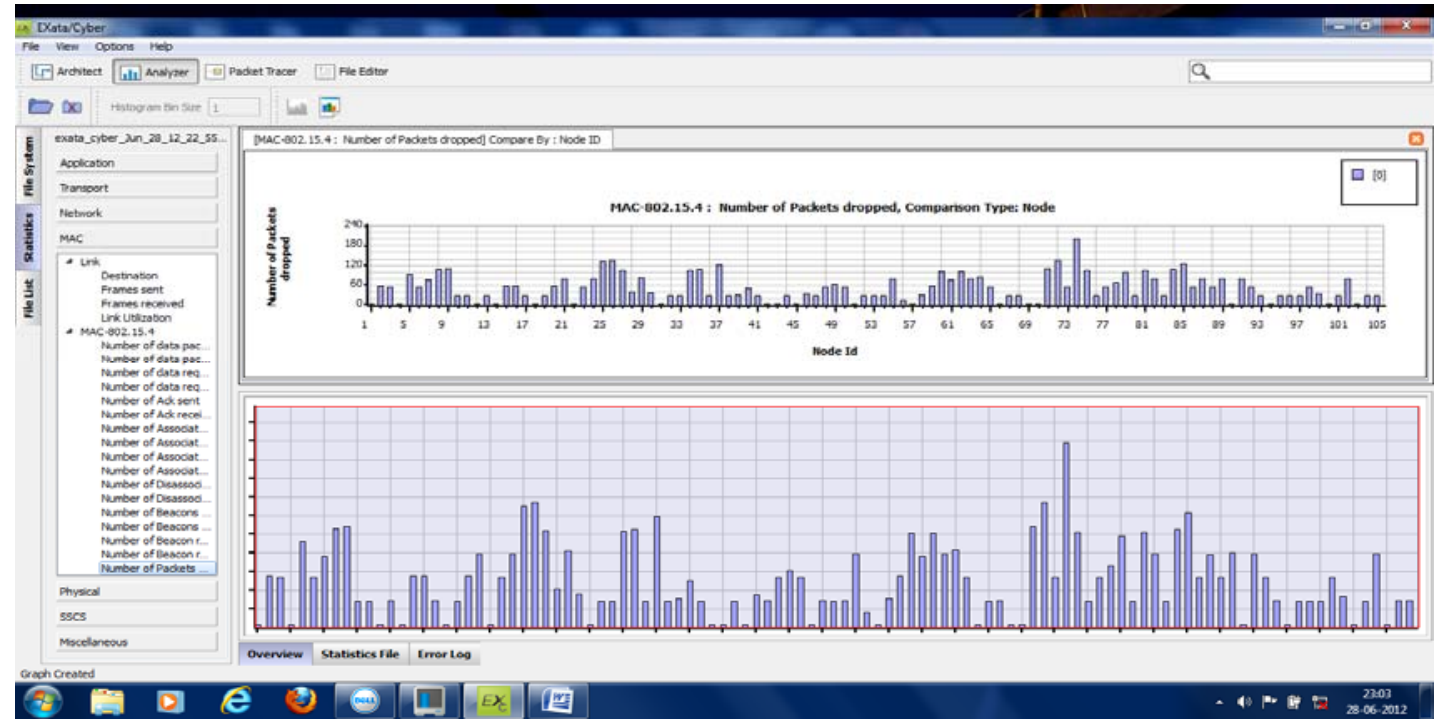

Fig7 : No. of pkt dropped in CL\& G 

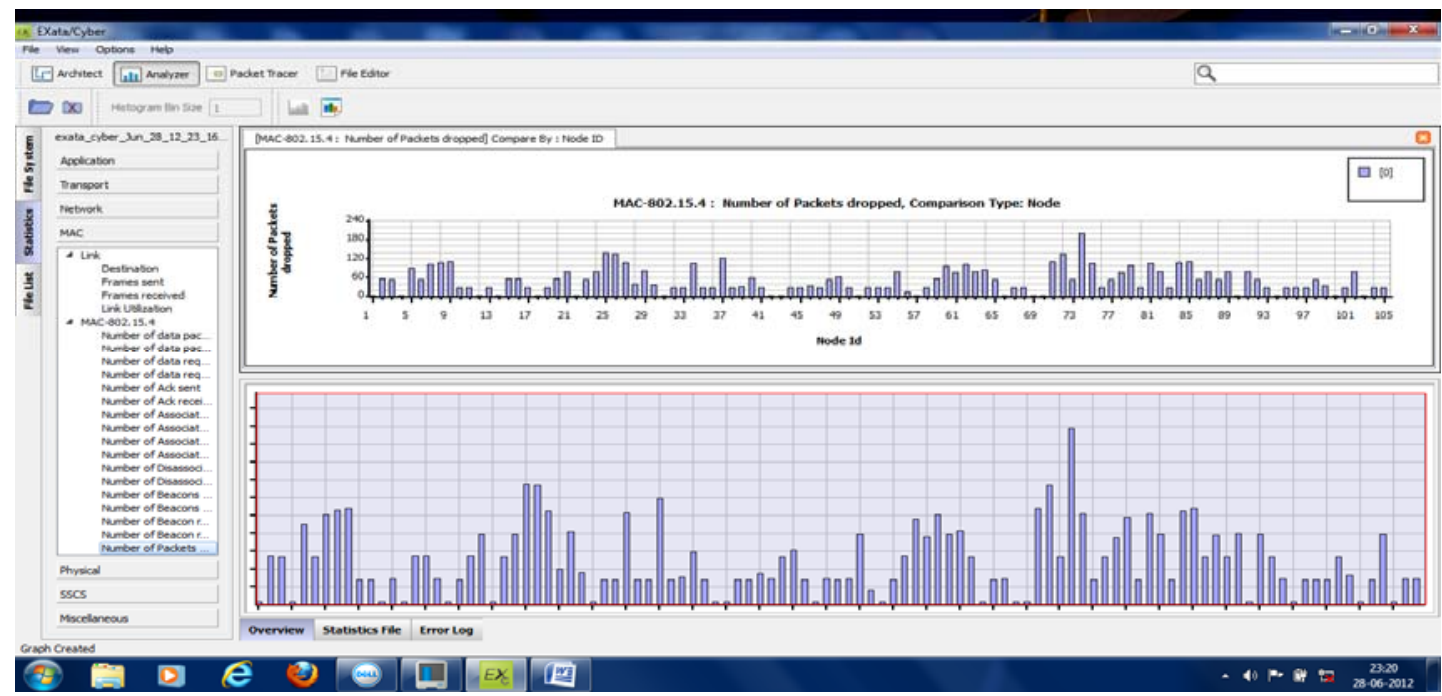

Fig8: No. of pkt dropped in CLG

\section{Performance Analysis for link utilization in CL \& G and CLG Approach}

In the result analysis of CBR server with cluster leader \& gateway , and Cluster leader gateway (CLG) the link utilization is better and effective in CLG approach shown in the Fig.9 and Fig.10

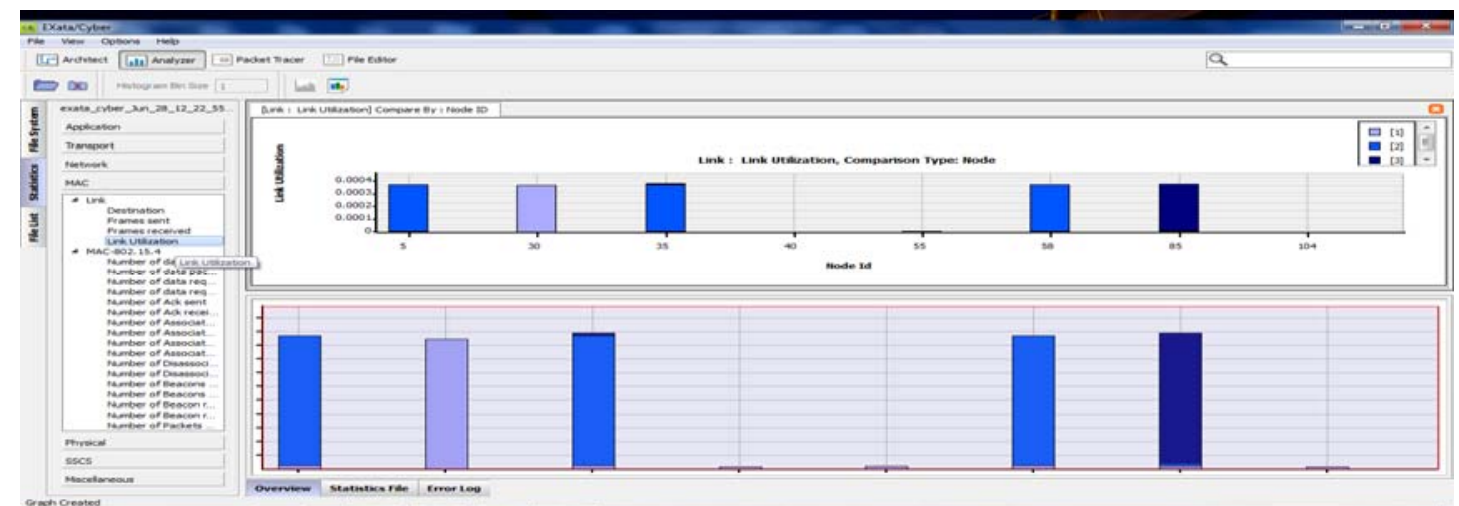

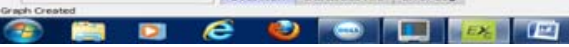

Fig9. Link utilization in CH \& G

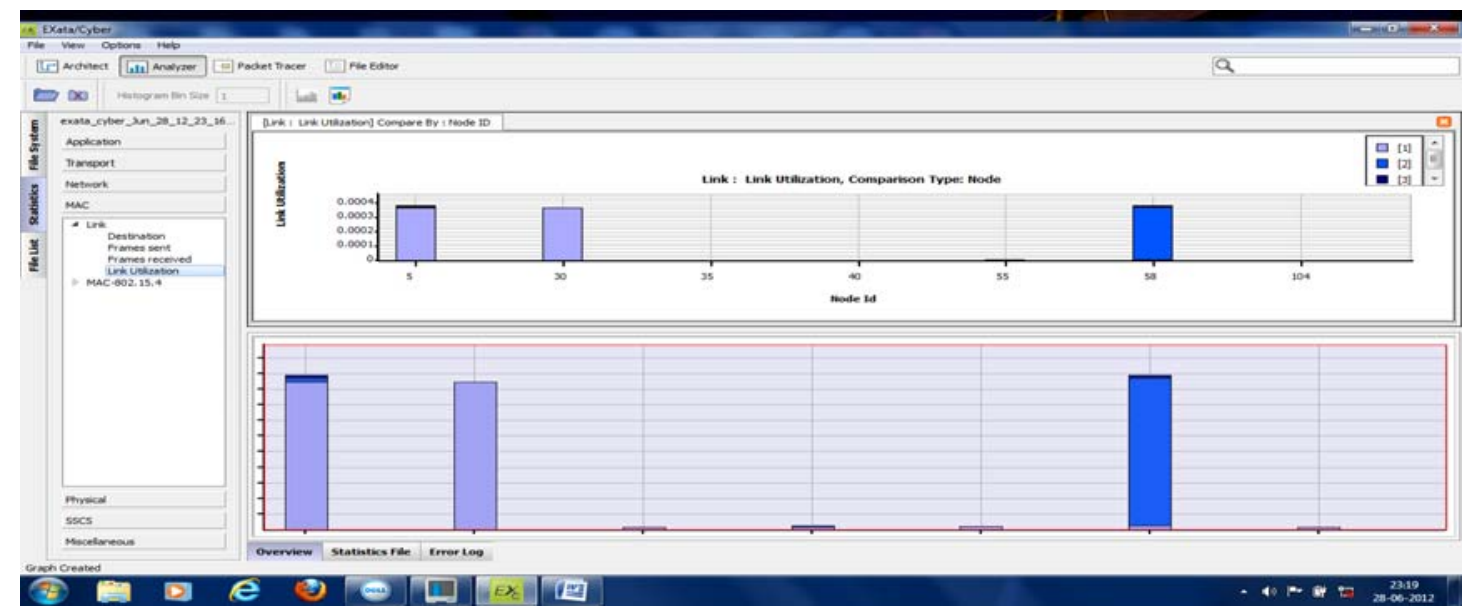

Fig10. Link utilization in CH G 


\section{CONCLUSION/FUTURE WORK}

Wireless mobile adhoc network is gaining popularity day by day because of its low cost and ease-of-use and in fact this is a next generation technology. In the given approach, we have reduced the election of cluster leader and election of gateway node separately with the novel CLG approach. Simulation analysis shows that CLG approach perform better for the parameters end to end delay, jitter, number of packets drop and link utilization. Hence by CLG approach overall performance of the network may be increased.

\section{ACKNOWLEDGMENT}

This work is supported by Graphic Era University, Dehradun (India) for providing research Lab and my mentor Dr. S.C Gupta (. Professor IIT Roorkee), and Dr. R. C Joshi (.Professor IIT Roorkee \& Chancellor Graphic Era University, Dehradun (India ).

\section{REFRENCES}

[1] Pin-Chuan Liu, Da-You Chen and Chin-Lin Hu,” Analysing The TCP Performance On Mobile Ad-Hoc Networks”, International Conference 2011.

[2] R.S.Shaji ,Rosaries ,F.Rajeshand Ramakrishnan, “A Novel Routing And Service Migration Scheme For Communication Among Heterogeneous Devices in Pervasive Environment”, “International Conference On Computer Communication And Electrical Technology", March 2011.

[3] Ismail Ghazi ShayebA et al.Survey of Clustering Schemes for Mobile Ad-Hoc Network MANET)American Journal of Scientific Research ISSN 1450-223X Issue 20(2011), pp.135-151 @ EuroJournals Publishing, Inc.2011

[4] Anzar Ahamd et al. "A Cluster Head Gateway Approach For Deciding The Cluster Head In Mobile Adhoc Network" ।"International Conference on Computer Networks and Securityl" World Academic of Science Engineering and Technology(WASET) Journal in Singapore December 2011 ICCNS 2010

[5] P.Krishna, Chatterjee, N.H Vaidya,\& D.K Pradhan,” A Cluster based Approach for routing in adhoc networks”, Proceeding of $2^{\text {nd }}$ USENIX Symposium on Mobile and Location Independent Computing.P.1 1995

[6] David B. Johnson and David A.Maltz, "Dynamic source routing in adhoc wireless networks". In Mobile Computing edited by Tomasz Imielinski and Hank Korth, chapter 5, pages 153-181. Kluwer Academic Publishers.

[7] David B. Johnson and David A. Maltz, "Protocols for adaptive wireless and mobile computing". In IEEE Personal Communications, 3(1), February 1996.

[8] Meysam Alikhany and Mohdi Abadi , "A Dynamic Clustering Based Approach For Anomaly Detection in AODV Based MANETS", "International Symposium On Computer Networks and Distributed Systems”,2011.

[9] HiuLi, Xiaoguang Zhang,Ying Liu , "Energy Efficient Routing Based on Ant Colony Algorithm In Mine Equipment Monitoring”,

[10] S.S.Kulkarni, G.R.Dattatreya, H.Martinez and R.Soto "Adaptive Control of Heterogeneous Ad Hoc Networks", Wireless Communications and Mobile Computing, Vol-4, 963-975, August 2004.

[11] Ram Ramanathan and Jason Redi, "A Brief Overview of Ad Hoc Networks - Challenges and Directions”, IEEE Communications Magazine 50th Anniversary Commemorative Issue, 20-22, May 2002.

[12] M.Q.Rafiq, S.R Chauduri, S.Kumar, S.Husain "Deciding the Cluster Size on Behalf of Co- channel Interference Ratio for the Omni Directional Antenna in Mobile Computing”ISTE day conference New Delhi Chapter $31^{\text {st }}$ May \& $1^{\text {st }}$ June20,08.

[13] I. Er and W. Seah, "Mobility-based d-hop clustering algorithm for mobile ad hoc networks," in Wireless Communications and Networking Conference, 2004. WCNC. 2004 IEEE, vol. 4, 2004, pp. 2359-2364.

[14] B.Das, E. Sivakumar,V Bhargavan,”Routing in backbone network using a virtual backbone”, Proceeding of $6^{\text {th }}$ International Conference on computer communication and network (IC3N'97),1997

[15] B.Das,V Bhargava, "Routing in adhoc network using a spine”, IEEE International Conference on computer \& communication network (ICC'97),213.

[16] J. Wu and J. Cao, "Connected k-hop clustering in ad hoc networks," in ICPP '05: Proceedings of the 2005 International Conference on Parallel Processing (ICPP’05). Washington, DC, USA: IEEE Computer Society,20014, pp. 373-380.

[17] Dharma Prakash Agrawal and Qing- An Zeng,” Introduction to Wireless and Mobile System” University Cincinnati.

[18] L. Wang and S. Olariu, "Cluster maintenance in mobile ad-hoc networks, "Cluster Computing, vol. 8, no. 2-3,pp. 111-118, 2015.

\section{AUTHOR PROFILE}

Anzar Ahmad is presently working as an Associate Professor in the department of Electronics \& Communication Engineering at the Graphic Era University, Dehradun, India. He did his Bachelor of Engineering (Electronics \& Communication Engg) degree from Jamia Millia Islamia University New Delhi in 1995. He did M. Tech (Microwave and optical Communication) from RVD University, Rajasthan in 2007 and $\mathrm{PhD}$ in Wireless Mobile Adhoc networks from Graphic Era University, Dehradun. He has worked as a cosupervisor on a project title," Scheduling Algorithm for Tactical Multi-hop Mobile Ad-hoc Network” funded by DEAL (Defence Electronics Application Lab), Dehradun. He has published 22 research papers in various reputed national and international Journals/conferences. He has 18 years of experience in teaching and has taught several core subjects in M.Tech as well as in B.Tech like Advance Wireless communication, Telecommunication Switching, Data Communication Network, CDMA system. His research area is Mobile Adhoc Network (MANET) and Wireless Communication. He has done training on wireless mobile communication system (GSM). 